\title{
The tree-shrub vegetation in rocky outcrop cerrado areas in Goiás State, Brazil
}

\author{
TASSIANA REIS RODRIGUES DOS SANTOS ${ }^{1,2,5}$, JOSÉ ROBERTO RODRIGUES PINTO ${ }^{3}$, \\ EDDIE LENZA ${ }^{4}$ and HENRIQUE AUGUSTO MEWS ${ }^{1}$
}

(received: March 29, 2011; accepted: May 31, 2012)

\begin{abstract}
The tree-shrub vegetation in rocky outcrop cerrado areas in Goiás State, Brazil). We describe the floristic composition of the tree-shrub vegetation in 10 areas of rocky outcrop cerrado in Goiás State, Brazil. Ten $20 \times 50 \mathrm{~m}$ plots (totaling $1 \mathrm{ha}$ ) were established and all of the individuals with diameters at $30 \mathrm{~cm}$ above soil level $\left(\mathrm{DB}_{30}\right) \geq 5 \mathrm{~cm}$ were included in the sampling. Comparative analyses of the flora were realized using similarity indices (Sørensen and Czekanowski), classification analysis (TWINSPAN), and the Mantel test. A total of 13,041 tree-shrub individuals were sampled, distributed among 219 species, 129 genera and 55 families. Fabaceae was the most well-represented family, followed by Myrtaceae, Melastomataceae, Vochysiaceae, Malphigiaceae, and Rubiaceae. Fully $42.3 \%$ of the comparisons evaluated by the Sørensen index were $>0.50$, while all the values were $<0.50$ for the Czekanowski index, with the exception of Jaragua and Mara Rosa areas. The TWINSPAN classification generated four divisions and, in general, only the differences in the size of the population were responsible for the groupings. The Mantel test indicated that there was no relationship between floristic similarity and the distances between the areas $(\mathrm{r}=0.32, P=0.05)$. It therefore appears that the areas of rocky outcrop cerrado in Goiás State are relatively floristically homogeneous and that they are principally distinguished by the differences in the sizes of the populations of their dominant species, and the presence of exclusive species in certain areas.
\end{abstract}

Key words - biodiversity, Cerrado biome, floristic composition, floristic similarity, rocky outcrops

\section{INTRODUCTION}

The Cerrado (Brazilian cerrado) biome originally occupied approximately 2,000,000 $\mathrm{km}^{2}(22 \%)$ of the country (Oliveira Filho \& Ratter 2002, Ribeiro \& Walter 2008), but its area has been reduced over the years to only $60.5 \%$ of its original extent (Sano et al. 2009). The diversified phytophysiognomic mosaic of Cerrado includes open field formations characterized by herbaceous plants (principally grasses), cerrado formations characterized by grasses with scattered and thin trees and sub-arboreal plants, and forest formations dominated by arboreal species (Ribeiro \& Walter 2008). According to Sano et al. (2009), approximately $61 \%$ of the natural areas of Cerrado are cerrado formations, $32 \%$ are forest formations, while $7 \%$ are open herbaceous areas.

One of the principal phytophysiognomic types of the biome is cerrado sensu stricto, the category classified

1. Programa de Pós-Graduação em Ciências Florestais, Universidade de Brasília - UnB, Caixa Postal 04357, 70 919-970 Brasília, DF, Brazil.

2. Current address: Programa de Pós-Graduação em Botânica, UnB, Caixa Postal 04457, 70 919-970 Brasília, DF, Brazil.

3. Universidade de Brasília, Departamento de Engenharia Florestal, Caixa Postal 04357, 70 919-970 Brasília, DF, Brazil.

4. Universidade do Estado do Mato Grosso - Unemat, Departamento de Ciências Biológicas, Caixa Postal 08, 78 690-000 Nova Xavantina, MT, Brazil.

5. Corresponding author: tassianatxu@hotmail.com as cerrado, which demonstrates well-defined arboreal and shrub-herbaceous layers (Ribeiro \& Walter 2008) with arboreal coverage varying from $10 \%$ to $60 \%$ (Eiten 1992). According to Ribeiro \& Walter (2008), cerrado sensu stricto can be divided into four subtypes depending on the density of the tree-shrub component and local environmental conditions: dense cerrado, typical cerrado locally called cerrado típico, thin cerrado, and rocky outcrop cerrado locally called "cerrado rupestre".

Rocky outcrop cerrados generally occurs in environments with rock outcrops (Ribeiro \& Walter 2008) with predominantly shallow, poorly evolved and litholic neosols, with the A horizon lying directly on the rock surface, or with a very thin $\mathrm{C}$ horizon (Reatto et al. 2008). The root systems of the plants growing there cannot penetrate very deeply, so that woody individuals are predominately found in cracks and crevices that have accumulated larger volumes of substrate (Romero 2002, Reatto et al. 2008). The rocky outcrop cerrado vegetation is characterized by tree-shrub species with heights between 2 and $4 \mathrm{~m}$ covering between $5 \%$ and $20 \%$ of the surface; the subshrub-herbaceous layer is thin, with species typical of this vegetation type (Ribeiro \& Walter 2008).

Rocky outcrop cerrados cover approximately $6.6 \%$ of Goiás and are distributed throughout the state (Lima 2008), but only limited studies have been conducted in this important phytophysiognomy of the Cerrado biome (Manoel 1999, Amaral et al. 2006, Miranda et al. 
2007, Moura et al. 2007, 2010, Pinto et al. 2009, Lima et al. 2010), and is still little-known and incompletely explored scientifically. Studies of floristic composition furnish basic information for understanding different plant formations and are essential for subsequent detailed studies (Van den Berg \& Oliveira Filho 2000).

We describe here the floristic composition of the treeshrub vegetation in 10 areas of rocky outcrop cerrado in Goiás State, Brazil, present a checklist of the species that can be used to evaluate the contributions of the different taxa and the floristic composition of these environments, and evaluate the floristic similarities between the areas surveyed. To that end, we have assumed that the rocky outcrop cerrado is a differentiated environment, principally in relation to its edaphic conditions: poor and thin soils with low $\mathrm{pH}$ and high concentrations of exchangeable aluminum (Benites et al. 2003, Reatto et al. 2008) - and should therefore demonstrate a unique floristic composition. In addressing these objectives, the following questions were formulated: What treeshrub species grow in the rocky outcrop cerrado areas of Goiás State? Does the rocky outcrop cerrado in that area contain an elevated floristic richness of tree-shrub species? Are the study areas floristically similar? Is the tree-shrub vegetation of this phytophysiognomy composed of generalist species or species specialized for a specific habitat?

\section{MATERIAL AND METHODS}

Study area - The current study was undertaken in 10 areas of rocky outcrop cerrado in Goiás State $(\mathrm{GO})$, in the central western region of Brazil (figure 1) $\left(12^{\circ} 23^{\prime} 46^{\prime \prime}\right.$ to $19^{\circ} 29^{\prime} 42^{\prime \prime} \mathrm{S}$ and $45^{\circ} 58^{\prime} 36^{\prime \prime}$ to $\left.53^{\circ} 14^{\prime} 53^{\prime \prime} \mathrm{W}\right)$. The regional climate is type Aw by the Köppen classification system, and is characterized by dry winters and rainy summer seasons, with annual average temperatures varying from $18{ }^{\circ} \mathrm{C}$ to $26^{\circ} \mathrm{C}$ and annual precipitation rates oscillating between

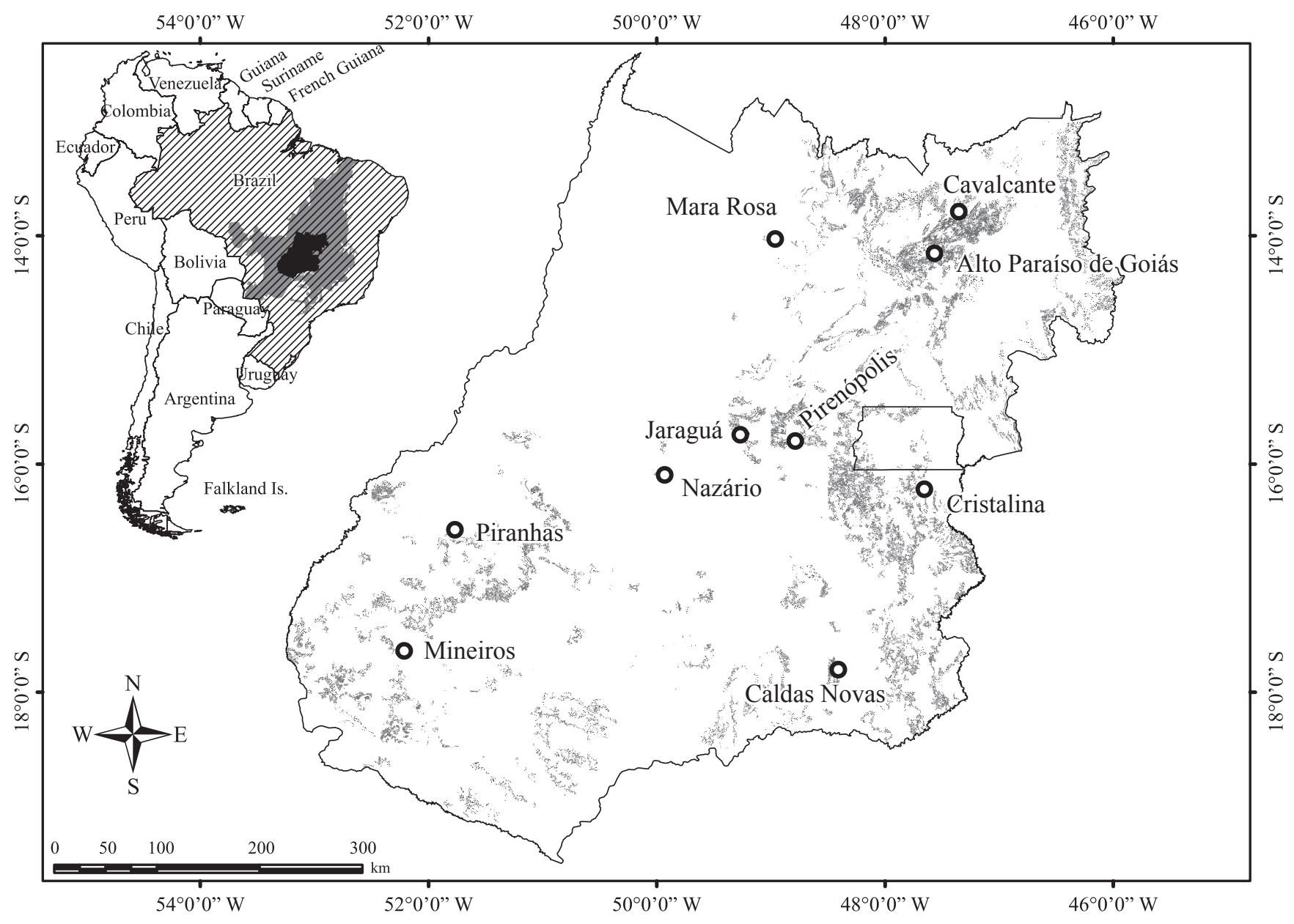

Figure 1. Location of the rocky outcrop cerrado areas sampled $(\bullet)$ in the Goiás State $(\mathrm{GO})$, where: hatched = Brazil; dark grey $=$ Cerrado biome; black = Goiás State; light grey in Goiás = regions with potential occurrence of rocky outcrop cerrado, according to Lima (2008). 
1,200 and 2,500 $\mathrm{mm}$ (Sieg 2009). The highest elevation in the state is Morro Alto in the region of Chapada dos Veadeiros (approximately 1,921 m), while the lowest altitudes are found in the Araguaia region (approximately $182 \mathrm{~m}$ ) (Sieg 2009) (table 1). Litholic neosols, upon which rocky outcrop cerrado vegetation normally develops, occupy approximately $8 \%$ of the state (Sano et al. 2006).

According to Lima (2008), rocky outcrop cerrado areas cover approximately $6.6 \%$ of Goiás State. These areas occur throughout the state, with greater frequency in the northeast in the micro-regions of Chapada dos Veadeiros, in the northern section of Brasília and Porangatu neighborhood. The present study surveyed areas in different regions where rocky outcrop cerrado occurs in Goiás State: in the northeast (three areas), southeast (two areas), southwest (two areas), and central region (three areas). The northwestern region of the state was excluded as there are few records of rocky outcrop cerrado there (Lima 2008).

Table 1. Spatial characteristics of the 10 rocky outcrop cerrado areas surveyed in Goiás State, Brazil.

\begin{tabular}{|c|c|c|c|c|c|c|}
\hline Areas & $\begin{array}{l}\text { Latitude } \\
\text { (S) }\end{array}$ & $\begin{array}{l}\text { Longitude } \\
\text { (W) }\end{array}$ & $\begin{array}{l}\text { Altitude } \\
(\mathrm{m})\end{array}$ & $\begin{array}{l}\text { Precipitation } \\
\quad(\mathrm{mm})\end{array}$ & $\begin{array}{l}\text { Average temperature } \\
\qquad\left({ }^{\circ} \mathrm{C}\right)\end{array}$ & Lithology \\
\hline Alto Paraíso de Goiás & $14^{\circ} 09^{\prime} 25^{\prime \prime}$ & $47^{\circ} 36^{\prime} 25^{\prime \prime}$ & 865 & 1,799 & 21.7 & quartzite \\
\hline Caldas Novas & $17^{\circ} 47^{\prime} 42^{\prime \prime}$ & $48^{\circ} 39^{\prime} 50^{\prime \prime}$ & 670 & 1,386 & 23.0 & sandstone \\
\hline Cavalcante & $13^{\circ} 47^{\prime} 29^{\prime \prime}$ & $47^{\circ} 23^{\prime} 41^{\prime \prime}$ & 900 & 1,720 & 23.6 & sandstone \\
\hline Cristalina & $16^{\circ} 43^{\prime} 31^{\prime \prime}$ & $47^{\circ} 41^{\prime} 50^{\prime \prime}$ & 1,100 & 1,368 & 20.8 & quartzite \\
\hline Jaraguá & $15^{\circ} 47^{\prime} 33^{\prime \prime}$ & $49^{\circ} 20^{\prime} 35^{\prime \prime}$ & 1,400 & 1,658 & 23.6 & sandstone \\
\hline Mara Rosa & $14^{\circ} 01^{\prime} 53^{\prime \prime}$ & $49^{\circ} 00^{\prime} 11^{\prime \prime}$ & 650 & 1,849 & 24.9 & sandstone \\
\hline Nazário & $16^{\circ} 41^{\prime} 00^{\prime \prime}$ & $49^{\circ} 45^{\prime} 33^{\prime \prime}$ & 650 & 1,467 & 23.4 & sandstone \\
\hline Piranhas & $16^{\circ} 26^{\prime} 99^{\prime \prime}$ & $51^{\circ} 53^{\prime} 99^{\prime \prime}$ & 750 & 1,700 & 23.4 & granite \\
\hline Pirenópolis & $15^{\circ} 48^{\prime} 13^{\prime \prime}$ & $48^{\circ} 49^{\prime} 39^{\prime \prime}$ & 850 & 1,564 & 20.8 & sandstone \\
\hline Mineiros & $17^{\circ} 39^{\prime} 53^{\prime \prime}$ & $52^{\circ} 16^{\prime} 39^{\prime \prime}$ & 800 & 1,642 & 22.8 & basalt \\
\hline
\end{tabular}

Vegetation survey - We established ten $20 \times 50 \mathrm{~m}$ plots, totaling 1 ha for each site, in each of the 10 areas inventoried, following the protocols established for the Network of Permanent Plots in the Cerrado and Pantanal Biomes (Felfili et al. 2005). All the individuals with diameter $30 \mathrm{~cm}$ above soil level $\left(\mathrm{DB}_{30}\right) \geq 5 \mathrm{~cm}$ were considered tree-shrub plants and included in the sampling, as recommended by Felfili et al. (2005). The botanical identifications were made in the field and by consulting the specific appropriate literature and herbaria samples stored at the Universidade de Brasília and Instituto Brasileiro de Geografia e Estatística, as well as specialists. The APG III (2009) classification system was adopted for the botanical families. The revision and updating of the taxa names was performed following Forzza et al. (2010). The classification of the species as well as their habits (the characteristics or appearances of plants: vines, subshrubs, shrubs, treelets, and trees) followed Mendonça et al. (2008).

Data analysis - The qualitative Sørensen index (Brower \& Zar 1977) and the quantitative Czekanowski index (Kent \& Coker 1992) were used to analyze the floristic similarities among the 10 areas inventoried. According to Kent \& Coker (1992), the Sørensen and Czekanowski index values vary between zero and one, with values greater than 0.5 indicating high similarities between the communities. Additionally, the Mantel test (Legendre \& Legendre 1998), using the PCORD 4 software program (McCune \& Mefford 1999), was used to evaluate if the floristic similarity values generated by the Czekanowski index were related to the geographical distances between the areas. To evaluate the significance of the Mantel test, 1,000 random Monte Carlo permutations were run. TWINSPAN (Kent \& Coker 1992) analysis was used to identify divisions and grouping patterns among areas based on floristic characteristics, which indicated and identified the preferential and indicator species of each area.

\section{RESULTS AND DISCUSSION}

A total of 13,041 tree-shrub individuals were surveyed, distributed among 219 species, 129 genera, and 55 botanical families; 196 (84.49\%) of these taxa were identified to the species level, $17(7.76 \%)$ to the genus level, five $(2.28 \%)$ to the family level; one plant remained without definitive indication (table 2). In terms of their habits, $113(57.65 \%)$ species were considered trees, $24(12.24 \%)$ shrubs, $18(9.18 \%)$ treelets, 15 (7.65\%) trees/shrubs, $13(6.63 \%)$ treelets/shrubs, six $(3.06 \%)$ subshrubs, five $(2.65 \%)$ trees/treelets, one $(0.51 \%)$ shrub/subshrub, and one $(0.51 \%)$ tree/vine.

According to Mendonça et al. (2008), the Cerrado biome as a whole contains 11,627 species of vascular phanerogams, 4,346 of which are considered tree-shrub. As such, the species recorded in the 10 rocky outcrop cerrado areas sampled here represent approximately 
Table 2. Tree-shrub species $\left(\mathrm{DB}_{30} \geq 5 \mathrm{~cm}\right)$ with their respective densities, sampled in 10 areas of rocky outcrop cerrado (ten $20 \times 50 \mathrm{~m}$ plots) in Goiás State, Brazil. The species are arranged in alphabetical order of their botanical families, where: $*$ = widely distributed species in the Cerrado biome (Ratter et al. 2003); ** = specialist species in a specific habitat (Munhoz \& Proença 1998, Ratter et al. 2000, Silva et al. 2001, Ribeiro \& Walter 2008, Pinto et al. 2009); $\square=$ forest species; $0=$ not abundant regionally. The regions in which the species were found are represented by the letters NE (northeast), SE (southeast), SW (southwest) and CE (central).

\begin{tabular}{|c|c|c|}
\hline Family/species & Habit & Number of areas \\
\hline \multicolumn{3}{|l|}{ ANACARDIACEAE } \\
\hline Anacardium occidentale L. & tree & $\mathrm{NE}(1) ; \mathrm{SW}(1) ; \mathrm{CE}(2)$ \\
\hline Astronium fraxinifolium Schott* ${ }^{*}$ & tree & $\mathrm{NE}(1) ; \mathrm{SW}(1)$ \\
\hline Tapirira guianensis Aubl. $\square ०$ & tree & NE (1) \\
\hline \multicolumn{3}{|l|}{ ANNONACEAE } \\
\hline Annona coriacea Mart.* & tree/shrub & NE (1); SE (2); SW (1) \\
\hline Annona crassiflora Mart. & tree & SW (1) \\
\hline Guatteria sellowiana Schltdl. $\square$ & tree & $\mathrm{CE}(1)$ \\
\hline Xylopia aromatica (Lam.) Mart.* & tree & NE (1); SE (2); SW (1); CE (2) \\
\hline \multicolumn{3}{|l|}{ APOCYNACEAE } \\
\hline Aspidosperma macrocarpon Mart. & tree & NE (1); SE (2); SW (2); CE (3) \\
\hline Aspidosperma multiflorum A.DC. & tree & NE (2) \\
\hline Aspidosperma ramiflorum Müll. Arg. $\square \circ$ & tree & SE (1) \\
\hline Aspidosperma subincanum Mart. ex A.DC. $\square$ & tree & SW (1) \\
\hline Aspidosperma tomentosum Mart.* & tree & NE (2); SE (2); SW (2); CE (3) \\
\hline Aspidosperma $\mathrm{sp}$. & - & $\mathrm{CE}(1)$ \\
\hline Hancornia speciosa Gomes* & tree & NE (2); SE (2); SW (2); CE (2) \\
\hline Himatanthus obovatus (Müll. Arg.) Woodson* & tree & NE (1); SE (1); CE (2) \\
\hline \multicolumn{3}{|l|}{ AQUIFOLIACEAE } \\
\hline Ilex congesta $\mathrm{H} . \mathrm{W} . \mathrm{Li}$ & shrub & NE (1) \\
\hline Ilex conocarpa Reissek & tree & $\mathrm{CE}(1)$ \\
\hline Ilex sp. & - & SW (1) \\
\hline \multicolumn{3}{|l|}{ ARALIACEAE } \\
\hline Schefflera burchellii (Seem.) Frodin \& Fiaschi ○ & tree & $\mathrm{NE}(1)$ \\
\hline Schefflera macrocarpa (Cham. \& Schltdl.) Frodin & tree & $\mathrm{NE}(2) ; \mathrm{SE}(2) ; \mathrm{CE}(3)$ \\
\hline $\begin{array}{l}\text { Schefflera vinosa (Cham. \& Schltdl.) Frodin \& } \\
\text { Fiaschi } \bigcirc\end{array}$ & tree & SW (1) \\
\hline \multicolumn{3}{|l|}{ ARECACEAE } \\
\hline Acrocomia aculeata (Jacq.) Lodd. ex Mart. $\square$ & tree & SW (1) \\
\hline Butia sp. & - & SW (1) \\
\hline Syagrus comosa (Mart.) Mart. & shrub & NE (2); SW (1); CE (1) \\
\hline Syagrus flexuosa (Mart.) Becc. & shrub & NE (3); SE (1); SW (1) \\
\hline \multicolumn{3}{|l|}{ ASTERACEAE } \\
\hline Eremanthus glomerulatus Less. & tree & NE (2); SE (2); CE (2) \\
\hline Eremanthus goyazensis (Gardner) Sch. Bip. & treelet & NE (1); SE (1) \\
\hline Piptocarpha rotundifolia (Less.) Baker & treelet & SW (1) \\
\hline Wunderlichia cruelsiana Taub.** & tree & NE (2); CE (2) \\
\hline Wunderlichia mirabilis Riedel ex Baker ** & treelet & NE (1); SE (2); SW (1); CE (1) \\
\hline Asteraceae NI 10 & - & SE (1) \\
\hline Asteraceae NI 2 & - & SW (1) \\
\hline \multicolumn{3}{|l|}{ BIGNONIACEAE } \\
\hline Handroanthus ochraceus (Cham.) Mattos* & tree & NE (1); SE (2); CE (3) \\
\hline Handroanthus serratifolius (Vahl) S.O.Grose & tree & NE (1); SE (1); SW (1); CE (2) \\
\hline $\begin{array}{l}\text { Tabebuia aurea (Silva Manso) Benth. \& Hook.f. } \\
\text { ex S.Moore* }\end{array}$ & tree & NE (1); SW (1); CE (2) \\
\hline Tabebuia sp. & - & SE (1) \\
\hline
\end{tabular}


continuation

\begin{tabular}{|c|c|c|}
\hline Family/species & Habit & Number of areas \\
\hline \multicolumn{3}{|l|}{ CALOPHYLLACEAE } \\
\hline Kielmeyera coriacea Mart. \& Zucc.* & tree & NE (3); SE (2); SW (2); CE (3) \\
\hline Kielmeyera lathrophyton Saddi & tree & $\mathrm{NE}(2)$ \\
\hline Kielmeyera rubriflora Cambess. & shrub & NE (2); SW (1); CE (1) \\
\hline Kielmeyera speciosa A.St.-Hil. & tree & NE (1); SE (2); SW (1); CE (3) \\
\hline \multicolumn{3}{|l|}{ CARYOCARACEAE } \\
\hline Caryocar brasiliense Cambess.* & tree & NE (2); SE (1); SW (2); CE (2) \\
\hline Caryocar cuneatum Wittm. & tree & NE (1) \\
\hline \multicolumn{3}{|l|}{ CELASTRACEAE } \\
\hline Plenckia populnea Reissek & tree & NE (2); SE (2); SW (1); CE (3) \\
\hline Salacia crassifolia (Mart. ex Schult.) G.Don & tree/treelet & NE (2); SE (1); CE (2) \\
\hline Salacia elliptica (Mart. ex Schult.) G.Don $\square$ & tree & $\mathrm{SE}(1)$ \\
\hline \multicolumn{3}{|l|}{ CHRYSOBALANACEAE } \\
\hline Couepia grandiflora (Mart. \& Zucc.) Benth. ex Hook.f. & tree & NE (3); SE (1); SW (1) \\
\hline Hirtella glandulosa Spreng. ๑० & tree & NE (1) \\
\hline Licania humilis Cham. \& Schltdl. $\square$ & tree & SE (2) \\
\hline \multicolumn{3}{|l|}{ CLUSIACEAE } \\
\hline Clusia weddelliana Planch. \& Triana** & tree & NE (1); CE (1) \\
\hline \multicolumn{3}{|l|}{ COMBRETACEAE } \\
\hline Buchenavia tomentosa Eichler $\square$ & tree & SW (1) \\
\hline Terminalia argentea Mart. $\square$ & tree & SW (1) \\
\hline \multicolumn{3}{|l|}{ CONNARACEAE } \\
\hline Connarus suberosus Planch.* & shrub & NE (3); SE (2); SW (2); CE (2) \\
\hline Rourea induta Planch. & treelet & NE (2); SW (1); CE (1) \\
\hline \multicolumn{3}{|l|}{ DILLENIACEAE } \\
\hline Curatella americana L.* & tree & NE (1); SW (2) \\
\hline Davilla elliptica A.St.-Hil.* & tree & NE (2); SE (2); SW (2); CE (2) \\
\hline \multicolumn{3}{|l|}{ EBENACEAE } \\
\hline Diospyros burchellii Hiern & tree & NE (1); SW (1); CE (2) \\
\hline Diospyros sericea A.DC. $\square$ & tree & NE (1) \\
\hline Diospyros sp. $\circ$ & - & NE (1) \\
\hline \multicolumn{3}{|l|}{ ERICACEAE } \\
\hline Agarista chapadensis (Kin.-Gouv.) Judd & treelet & NE (1) \\
\hline \multicolumn{3}{|l|}{ ERYTHROXYLACEAE } \\
\hline Erythroxylum anguifugum Mart. ○ & treelet & $\mathrm{NE}(1)$ \\
\hline Erythroxylum daphnites Mart. $\square$ & treelet & NE (1); SW (1) \\
\hline Erythroxylum deciduum A.St.-Hil. & tree/treelet & NE (2); SW (1); CE (3) \\
\hline Erythroxylum engleri O.E.Schulz $\bigcirc$ & treelet & SW (1) \\
\hline Erythroxylum suberosum A.St.-Hil.* & treelet & NE (3); SE (1); SW (2); CE (3) \\
\hline Erythroxylum tortuosum Mart. & treelet & NE (1); SE (2); CE (1) \\
\hline Erythroxylum sp. 1 & - & $\mathrm{NE}(1)$ \\
\hline Erythroxylum sp. $2 \circ$ & - & NE (1) \\
\hline \multicolumn{3}{|l|}{ EUPHORBIACEAE } \\
\hline Alchornea triplinervia (Spreng.) Müll.Arg. $\square$ & tree & CE (1) \\
\hline Maprounea guianensis Aubl. $\square$ & tree & SE (1); CE (1) \\
\hline Euphorbiaceae NI & - & SW (1) \\
\hline \multicolumn{3}{|l|}{ FABACEAE } \\
\hline Acosmium sp. & - & SW (1) \\
\hline Andira paniculata Benth. & tree & NE (2); SE (1); SW (1); CE (1) \\
\hline Andira vermifuga Mart. ex Benth. & tree & NE (2); SE (1); CE (2) \\
\hline Bauhinia pulchella Benth. & shrub & NE (1) \\
\hline Bowdichia virgilioides Kunth* & tree & NE (2); SE (1); SW (2); CE (2) \\
\hline
\end{tabular}


continuation

\begin{tabular}{|c|c|c|}
\hline Family/species & Habit & Number of areas \\
\hline Chamaecrista orbiculata (Benth.) H.S.Irwin \& Barneby & treelet/shrub & NE (2); SE (1); SW (1); CE (2) \\
\hline $\begin{array}{l}\text { Chamaecrista pachyclada (Harms) H.S.Irwin \& } \\
\text { Barneby }\end{array}$ & subshrub & $\mathrm{NE}(1) ; \mathrm{CE}(1)$ \\
\hline Chamaecrista sp. 1 & - & NE (1) \\
\hline Chamaecrista sp. 2 & - & SW (1) \\
\hline Copaifera langsdorffii Desf. $\square$ & tree & SE (1); SW (1); CE (1) \\
\hline Copaifera oblongifolia Mart. & treelet/shrub & NE (2) \\
\hline Dalbergia miscolobium Benth. & tree & NE (1); SE (2); SW (1); CE (1) \\
\hline Dimorphandra mollis Benth.* & tree & NE (2); SE (1); SW (1); CE (1) \\
\hline Dipteryx alata Vogel $\square$ & tree & $\mathrm{SO}(1)$ \\
\hline Hymenaea stigonocarpa Mart. ex Hayne* & tree & NE (1); SE (2); SW (2); CE (3) \\
\hline Leptolobium dasycarpum Vogel* & tree/treelet & NE (2); SE (2); SW (2); CE (3) \\
\hline Luetzelburgia praecox (Harms ex Kuntze) Harms & tree & NE (1); SW (1) \\
\hline Machaerium acutifolium Vogel* & tree & NE (1); SW (1) \\
\hline Machaerium opacum Vogel & tree & NE (2); SE (1); CE (1) \\
\hline Mimosa claussenii Benth. & shrub & NE (2); SE (1) \\
\hline Mimosa decorticans Harms ex Glaz. & treelet & $\mathrm{SE}(1)$ \\
\hline Mimosa manidea Barneby & treelet & NE (1) \\
\hline Mimosa setosissima Taub.** & shrub & CE (1) \\
\hline Mimosa ulei Taub. & shrub & $\mathrm{NE}(1)$ \\
\hline Peltogyne confertiflora (Mart. ex Hayne) Benth. $\square$ & tree & NE (1); SE (1); SW (1); CE (2) \\
\hline Plathymenia reticulata Benth.* & tree & NE (1); SE (2); SW (2); CE (2) \\
\hline Platypodium elegans Vogel $\square$ & tree & SW (1); CE (1) \\
\hline Pterodon emarginatus Vogel $\square$ & tree & $\mathrm{NE}(1) ; \mathrm{SW}(1)$ \\
\hline Pterodon pubescens (Benth.) Benth. & tree & NE (1); SE (2); CE (2) \\
\hline Senna velutina (Vogel) H.S. Irwin \& Barneby & treelet/shrub & NE (1) \\
\hline Stryphnodendron polyphyllum Mart. & tree/shrub & SE (1); SW (1) \\
\hline Stryphnodendron rotundifolium Mart. ○ & tree & SW (1) \\
\hline Tachigali aurea Tul.* & tree & NE (1); SW (1) \\
\hline Tachigali vulgaris L.G.Silva \& H.C.Lima & tree & NE (3); SE (2); SW (1); CE (2) \\
\hline Vatairea macrocarpa (Benth.) Ducke* & tree & NE (2); SE (1); SW (2); CE (2) \\
\hline \multicolumn{3}{|l|}{ HUMIRIACEAE } \\
\hline Humiria balsamifera Aubl. $\square$ & tree & NE (1) \\
\hline \multicolumn{3}{|l|}{ ICACINACEAE } \\
\hline Emmotum nitens (Benth) Miers $\square$ & tree & NE (2); SE (1); SW (1) \\
\hline \multicolumn{3}{|l|}{ LAMIACEAE } \\
\hline Aegiphila lhotskiana Cham. & tree/shrub & SE (2); SW (2); CE (2) \\
\hline Hyptis pachyphylla Epling** & shrub & $\mathrm{NE}(1)$ \\
\hline \multicolumn{3}{|l|}{ LAURACEAE } \\
\hline Aniba heringeri Vattimo $\square ०$ & tree & NE (1) \\
\hline Endlicheria paniculata (Spreng.) J.F.Macbr. $\square$ & tree & SE (1) \\
\hline Mezilaurus crassiramea (Meisn.)Taub. ex Mez ○ & tree & SW (1) \\
\hline Ocotea pomaderroides (Meisn.) Mez $\square$ & tree & NE (1); CE (1) \\
\hline \multicolumn{3}{|l|}{ LOGANIACEAE } \\
\hline Antonia ovata Pohl & shrub & SW (1) \\
\hline Strychnos pseudoquina A.St.-Hil. & tree & SW (1) \\
\hline \multicolumn{3}{|l|}{ LYTHRACEAE } \\
\hline Lafoensia pacari A.St.-Hil.* & tree/shrub & NE (1); SE (2); SW (1); CE (2) \\
\hline \multicolumn{3}{|l|}{ MALPHIGIACEAE } \\
\hline Banisteriopsis latifolia (A.Juss.) B.Gates & treelet & NE (3); SW (1); CE (3) \\
\hline Byrsonima basiloba A.Juss. & shrub & SW (1) \\
\hline Byrsonima coccolobifolia Kunth* & tree & NE (3); SE (2); SW (2); CE (2) \\
\hline
\end{tabular}


continuation

\begin{tabular}{|c|c|c|}
\hline Family/species & Habit & Number of areas \\
\hline Byrsonima crassifolia (L.) Kunth $\circ$ & tree/shrub & SE (1) \\
\hline Byrsonima intermedia A.Juss. & tree/shrub & NE (1) \\
\hline Byrsonima pachyphylla A.Juss.* & treelet/shrub & NE (3); SE (2); SW (1); CE (3) \\
\hline Byrsonima verbascifolia (L.) Rich. ex Juss.* & tree & NE (1); SW (1); CE (1) \\
\hline Byrsonima sp. & - & SW (1) \\
\hline Heteropteris byrsonimifolia A.Juss. & treelet/shrub & NE (3); SE (1); SW (1); CE (3) \\
\hline Tetrapterys microphylla Nied. $\circ$ & shrub & $\mathrm{NE}(1)$ \\
\hline \multicolumn{3}{|l|}{ MALVACEAE } \\
\hline Eriotheca gracilipes (K.Schum.) A.Robyns* $\square$ & tree & NE (2); SE (1); SW (2); CE (1) \\
\hline Eriotheca pubescens (Mart. \& Zucc.) Schott \& Endl. & treelet/shrub & NE (1); SE (2); SW (1); CE (1) \\
\hline Luehea candicans Mart. $\circ$ & tree & SW (1) \\
\hline Pseudobombax longiflorum (Mart. \& Zucc.) A. Robyns $\square$ & tree & NE (1); SE (1); CE (2) \\
\hline Pseudobombax tomentosum (Mart. \& Zucc.) Robyns $\square$ & tree & SW (1) \\
\hline \multicolumn{3}{|l|}{ MARCGRAVIACEAE } \\
\hline Norantea guianensis Aubl. & tree/vine & SW (2) \\
\hline $\begin{array}{l}\text { Schwartzia adamantium (Cambess.) Bedell ex } \\
\text { Giraldo-Cañas** }\end{array}$ & tree/treelet & NE (2); SE (2); SW (1); CE (2) \\
\hline \multicolumn{3}{|l|}{ MELASTOMATACEAE } \\
\hline Macairea radula (Bonpl.) DC. & treelet & $\mathrm{NE}(1) ; \mathrm{CE}(3)$ \\
\hline Miconia albicans (Sw.) Triana & treelet & NE (2); SE (2); SW (1); CE (1) \\
\hline Miconia burchellii Triana & tree & NE (2); SE (1); CE (1) \\
\hline Miconia ferruginata DC. & tree & NE (3); SE (2); SW (1); CE (2) \\
\hline Miconia irwinii Wurdack & tree & NE (1) \\
\hline Miconia leucocarpa DC.o & tree & CE (1) \\
\hline Miconia pepericarpa Mart. ex A.DC. $\bigcirc$ & tree/shrub & SE (1) \\
\hline Miconia rubiginosa (Bonpl.) DC. & shrub & NE (1); SE (1) \\
\hline Mouriri elliptica Mart. & treelet & SW (2) \\
\hline Tibouchina papyrus (Pohl) Toledo** & treelet/shrub & SW (1); CE (1) \\
\hline Tibouchina sp. 1 & - & NE (1) \\
\hline Tibouchina sp. 2 & - & SW (1) \\
\hline Tibouchina villosissima Cogn. $\circ$ & shrub & $\mathrm{CE}(1)$ \\
\hline \multicolumn{3}{|l|}{ MORACEAE } \\
\hline Brosimum gaudichaudii Trécul* & tree/shrub & NE (1); SE (1); CE (1) \\
\hline Ficus guaranitica Chodat $\square$ & tree & SE (1) \\
\hline Ficus sp. $\circ$ & - & SW (1) \\
\hline \multicolumn{3}{|l|}{ MYRISTICACEAE } \\
\hline Virola sebifera Aubl. $\square$ & tree & SE (1); SW (1) \\
\hline \multicolumn{3}{|l|}{ MYRSINACEAE } \\
\hline Myrsine guianensis (Aubl.) Kuntze & tree & NE (1); SE (1); SW (1); CE (1) \\
\hline \multicolumn{3}{|l|}{ MYRTACEAE } \\
\hline Blepharocalyx salicifolius (Kunth) O.Berg & tree/shrub & NE (2); SE (1) \\
\hline Eugenia aurata O.Berg & shrub & SW (1) \\
\hline Eugenia involucrata DC. $\square ०$ & tree/shrub & SE (1) \\
\hline Eugenia punicifolia (Kunth) DC. & shrub & NE (1); SW (1); CE (1) \\
\hline Myrcia bella Cambess. & shrub & NE (1); SE (1) \\
\hline Myrcia cordifolia O.Berg & shrub/subshrub & CE (1) \\
\hline Myrcia fenzliana O.Berg $\square$ & tree & CE (1) \\
\hline Myrcia multiflora (Lam.) DC. & tree/shrub & CE (1) \\
\hline Myrcia splendens (Sw.) DC. & tree & NE (1); SW (1) \\
\hline Myrcia tomentosa (Aubl.) DC. & tree & $\mathrm{SW}(1) ; \mathrm{CE}(1)$ \\
\hline Myrcia uberavensis O.Berg & shrub & SE (1) \\
\hline Myrcia variabilis DC. & subshrub & SE (1); SW (1) \\
\hline
\end{tabular}


continuation

\begin{tabular}{|c|c|c|}
\hline Family/species & Habit & Number of areas \\
\hline Myrcia sp. & - & NE (1) \\
\hline Myrtaceae NI & - & SW (1) \\
\hline Psidium myrsinoides O.Berg & shrub & NE (3); SE (2); CE (3) \\
\hline Psidium laruotteanaum O.Berg & treelet/shrub & SE (1); SW (1); CE (1) \\
\hline Psidium sp. $\circ$ & - & SE (1) \\
\hline Siphoneugena densiflora O.Berg $\square$ & tree & SE (1); CE (1) \\
\hline \multicolumn{3}{|l|}{ NOT IDENTIFIED } \\
\hline Not identified & - & SW (1) \\
\hline \multicolumn{3}{|l|}{ NYCTAGINACEAE } \\
\hline Guapira graciliflora (Schmidt) Lundell & tree & NE (2); SE (2); CE (3) \\
\hline Guapira noxia (Netto) Lundell & tree & $\mathrm{NE}(2) ; \mathrm{SE}(2) ; \mathrm{CE}(2)$ \\
\hline Neea theifera Oerst. & tree & NE (3); SE (2); SW (1); CE (2) \\
\hline \multicolumn{3}{|l|}{ OCHNACEAE } \\
\hline Ouratea glaucescens Engl. & tree/shrub & NE (2) \\
\hline Ouratea hexasperma (A.St.-Hil.) Baill.* & treelet/shrub & NE (3); SE (2); SW (1); CE (3) \\
\hline Ouratea spectabilis (Mart. ex Engl.) Engl. & tree & NE (1); SW (2); CE (1) \\
\hline \multicolumn{3}{|l|}{ OLACACEAE } \\
\hline Heisteria ovata Benth. & tree & SW (1); CE (1) \\
\hline \multicolumn{3}{|l|}{ OPILIACEAE } \\
\hline Agonandra brasiliensis Miers ex Benth. \& Hook.f. $\square$ & tree/shrub & NE (1); CE (2) \\
\hline \multicolumn{3}{|l|}{ PENTAPHYLACACEAE } \\
\hline Ternstroemia carnosa Cambess. & tree/shrub & NE (1) \\
\hline \multicolumn{3}{|l|}{ PRIMULACEAE } \\
\hline Cybianthus gardneri (A.DC.) G.Agostini $\square ०$ & tree & NE (1) \\
\hline \multicolumn{3}{|l|}{ PROTEACEAE } \\
\hline Roupala montana Aubl.* & tree & NE (2); SE (1); SW (1); CE (3) \\
\hline \multicolumn{3}{|l|}{ RUBIACEAE } \\
\hline Alibertia edulis (Rich.) A.Rich. ex DC. & shrub & NE (1); SW (1) \\
\hline Chomelia ribesioides Benth. ex A.Gray & shrub & SE (2); SW (1); CE (2) \\
\hline Cordiera concolor (Cham.) Kuntze & shrub & SE (2) \\
\hline Cordiera elliptica (Cham.) Kuntze & shrub & $\mathrm{NE}(1)$ \\
\hline Cordiera sessilis (Vell.) Kuntze $\square$ & treelet & NE (1); SW (1); CE (1) \\
\hline Ferdinandusa elliptica Pohl & tree & $\mathrm{NE}(2)$ \\
\hline Palicourea rigida Kunth & treelet/shrub & NE (3); SE (1); SW (1); CE (3) \\
\hline Rudgea viburnoides (Cham.) Benth. $\square$ & treelet/shrub & SE (1); SW (1) \\
\hline Tocoyena formosa (Cham. \& Schltdl.) K.Schum.* & treelet & NE (2); SE (2); CE (3) \\
\hline Rubiaceae NI & - & CE (1) \\
\hline \multicolumn{3}{|l|}{ SALICACEAE } \\
\hline Casearia sylvestris Sw.* & treelet/shrub & SE (2); SW (1) \\
\hline \multicolumn{3}{|l|}{ SAPINDACEAE } \\
\hline Magonia pubescens A.St.-Hil. & tree & SW (1) \\
\hline Matayba elaeagnoides Radlk. $\square \bigcirc$ & tree & SW (1) \\
\hline Matayba guianensis Aubl. $\square$ & tree & NE (1) \\
\hline \multicolumn{3}{|l|}{ SAPOTACEAE } \\
\hline Pouteria ramiflora (Mart.) Radlk.* & tree & NE (1); SE (2); SW (1); CE (2) \\
\hline Pouteria torta (Mart.) Radlk. & tree & SE (1) \\
\hline \multicolumn{3}{|l|}{ SIMAROUBACEAE } \\
\hline Simarouba versicolor A.St.-Hil. $\square$ & tree & NE (2); SE (2); SW (1); CE (2) \\
\hline \multicolumn{3}{|l|}{ SIPARUNACEAE } \\
\hline Siparuna guianensis Aubl. $\square$ & tree ou shrub & NE (1) \\
\hline \multicolumn{3}{|l|}{ SOLANACEAE } \\
\hline Solanum lycocarpum A.St.-Hil. ○ & treelet/shrub & SE (1) \\
\hline
\end{tabular}


continuation

\begin{tabular}{|c|c|c|}
\hline Family/species & Habit & Number of areas \\
\hline \multicolumn{3}{|l|}{ STYRACACEAE } \\
\hline Styrax ferrugineus Nees \& Mart. & tree/treelet & NE (1); SW (1); CE (1) \\
\hline \multicolumn{3}{|l|}{ SYMPLOCACEAE } \\
\hline Symplocos nitens (Pohl) Benth. $\square$ & tree & SE (1) \\
\hline \multicolumn{3}{|l|}{ URTICACEAE } \\
\hline Cecropia pachystachya Trécul $\square$ & tree & SW (1); CE (1) \\
\hline \multicolumn{3}{|l|}{ VELLOZIACEAE } \\
\hline Vellozia albiflora Pohl ○ & subshrub & SE (1) \\
\hline Vellozia squamata Pohl & subshrub & NE (3); SE (1); CE (2) \\
\hline Vellozia tubiflora (A.Rich.) Kunth** & subshrub & $\mathrm{NE}(2)$ \\
\hline Vellozia variabilis Mart. ex Schult.f.** & subshrub & NE (1); SW (1) \\
\hline \multicolumn{3}{|l|}{ VOCHYSIACEAE } \\
\hline Callisthene fasciculata Mart. $\square$ & tree & SW (1) \\
\hline Callisthene major Mart. $\square$ & tree & SW (1) \\
\hline Callisthene mollissima Warm. & tree & NE (2) \\
\hline Callisthene sp. & - & NE (1) \\
\hline Qualea grandiflora Mart.* & tree & NE (2); SE (2); SW (2); CE (2) \\
\hline Qualea multiflora Mart.* & tree & NE (1); SE (2); SW (2); CE (3) \\
\hline Qualea parviflora Mart.* & tree & NE (3); SE (2); SW (2); CE (3) \\
\hline Salvertia convallariodora A.St.-Hil.* & tree & NE (1); SW (1); CE (1) \\
\hline Vochysia elliptica Mart. & tree & NE (1); SE (1); SW (1); CE (1) \\
\hline Vochysia gardneri Warm. & tree & NE (3); SW (1) \\
\hline Vochysia rufa Mart. & tree & NE (2); SE (2); SW (1); CE (2) \\
\hline Vochysia thyrsoidea Pohl & tree & NE (1); SE (1); CE (1) \\
\hline
\end{tabular}

$5.03 \%$ of all of the tree-shrub species recorded for the entire biome. In terms of the cerrado (sensu lato), these species represent $9.55 \%$ of the recorded species, and for the rocky fields (sensu lato) they represent 17.39\%. This data indicates the high floristic representivity of the rocky outcrop cerrado vegetation in Goiás State in terms of the tree-shrub components of the cerrado formations of the Cerrado biome. Considering that rocky field environments represent only $6.6 \%$ of Goiás State (Lima 2008), their floristic representivity becomes even more apparent.

The local floristic richnesses varied from 61 (in the municipalities of Nazário, Piranhas and Pirenópolis) to 84 species (Mineiros). This range is considered normal among cerrado formations (cerrado sensu stricto), which usually have less than 100 species per hectare (Felfili et al. 2004). The municipality of Mineiros is located in the Paraná Guimarães eco-region proposed by Arruda et al. (2008), with both sedimentary and volcanic rock formations (such as basalt - which is rich in iron/magnesium minerals) (Reatto et al. 2008). According to Benites et al. (2007), areas with rock outcrops can demonstrate considerable edaphoenvironmental diversity, reflected in vegetation mosaics that are largely determined by the local topography and by micro-environmental aspects. As such, the regional environmental heterogeneity contributed to the high floristic richness observed in Mineiros.

In general, the tree-shrub species richnesses observed in each of the sampling areas was greater than that reported in other studies of rocky outcrop cerrado using similar survey methodologies. A study by Amaral et al. (2006) in the Federal District, for example, reported 51 species, and by Miranda et al. (2007) in the Serra Dourada-GO recorded 54 species, by Pinto et al. (2009) in Cocalzinho, GO $(65)$, by Moura et al. $(2007,2010)$ in Pirenópolis, GO (56 and 65 species respectively), and studies by Moura (2010) in the Rio Preto State Park, MG, in the Sete Cidades National Park-PI and in Cáceres, MT listed 42, 47 and 69 species respectively. The high richness values encountered in these areas demonstrate the necessity protecting rocky outcrop cerrado sites to ensure the preservation of their unique biodiversity. According to Benites et al. (2007), cerrado/rocky field environments are not suitable for agricultural and are therefore natural refuges for the regional flora and fauna - and should be considered priority areas for biodiversity conservation.

Among the 10 areas sampled, Fabaceae was the most representative plant family in terms of the 
numbers of species (35), followed by Myrtaceae (18), Melastomataceae (13), Vochysiaceae (12), Malphigiaceae (10), and Rubiaceae (10). The high representivities of these families were likewise observed in other studies of rocky outcrop cerrado sites in Brazil (Manoel 1999, Amaral et al. 2006, Moura et al. 2007, Pinto et al. 2009), as well as in cerrado areas sensu stricto (Marimon et al. 1998, Felfili et al. 2002, Assunção \& Felfili 2004), and it could be seen that the rocky field and typical cerrado areas of Goiás State do not differ greatly in terms of their most representative families. Nonetheless, Marcgraviaceae and Velloziaceae, two well-represented families in rocky outcrop cerrados in terms of their numbers of species, densities of individuals, and geographical distribution (table 2), are generally poorly represented in areas of deep soils of cerrado (Ratter et al. 2000, 2003, Bridgewater et al. 2004), thus defining certain floristic differences between typical and rocky outcrop cerrados in the state.

Goodland (1979) considered the family Fabaceae to be one of the most important plant groups in the Cerrado biome. Vochysiaceae was also one of the best represented families in the present study, and its species (e.g. Qualea grandiflora, Q. parviflora, Q. multiflora, Vochysia thyrsoidea and $V$. elliptica) are known to accumulate aluminum (Haridasan 2000). According to this same author, the families Melastomataceae (e.g. Miconia ferruginata and M. pohliana); Rubiaceae (Palicourea rigida) also accumulate this metal. It is important to note that all the species cited above and identified as aluminum accumulators were encountered in the present study.

The floras of the rocky outcrop cerrado areas analyzed were largely composed of species known from cerrado sensu stricto (cerrado) formations growing on deep soils $(74.40 \%)$ and forest species $(21.50 \%)$. Nine species $(4.10 \%)$ considered specialist to specific habitats (sensu Rabinowitz 1981) of rocky outcrop cerrado and that are restricted to rupestre environments were recorded: Clusia weddelliana, Schwartzia adamantium, Mimosa setosissima, Tibouchina papyrus, Wunderlichia mirabilis, W. cruelsiana, Hyptis pachyphylla (Ratter et al. 2000, Ribeiro \& Walter 2008, Pinto et al. 2009), Vellozia variabilis (Silva et al. 2001), and Vellozia tubiflora (Munhoz \& Proença 1998). Tibouchina papyrus is important because its distribution is restricted to Goiás State, occurring principally in Serra dos Pirineus, Serra Dourada, and Chapada dos Veadeiros (Munhoz \& Proença 1998). In spite of the fact that only a small number of specialist species restricted to specific habitats were encountered in the rocky outcrop cerrado areas studied here, a number of them (Wunderlichia cruelsiana, Schwartzia adamantium, Hyptis pachyphylla, Vellozia variabilis and V. tubiflora) were cited as being important in determining the structure of rocky outcrop cerrado sites in Caldas Novas-GO (Lima et al. 2010) and in Alto Paraíso de Goiás-GO (Lenza et al. 2011).

Of the 38 species cited by Ratter et al. (2003) as being widely distributed throughout the Cerrado biome, 37 were encountered in areas of rocky outcrop cerrado in Goiás State (table 2). According to Lima et al. (2010), these species can serve as sources of diaspores for cerrado sensu stricto areas on deep soils and can contribute to the recuperation of altered areas of that biome. Twenty-six species $(11.87 \%$ of the total) were considered of limited regional abundance in the 10 areas of rocky outcrop cerrado surveyed by us in Goiás State (table 2). A species was considered of limited regional abundance in the present study if only one individual was encountered in only one of the areas inventoried. Among all the species recorded in rocky outcrop cerrado areas in Goiás State, nine $(4.10 \%)$ were recorded in less than three $(<10 \%)$ of the 376 areas of Cerrado studied by Ratter et al. (2003) throughout Brazil. Among these species, only two (Erythroxylum anguifugum and Miconia pepericarpa) were found among the species considered to be of limited regional abundance in the present study, reinforcing the importance of rocky outcrop cerrado sites in the preservation of species demonstrating low general abundance as well as those that are specialists in specific habitats.

The floristic similarities of the 10 survey areas as calculated by the Sørensen index varied from 0.18 to 0.71 (table 3). Values $>0.50$ (indicating high similarities between areas) (Kent \& Coker 1992), were observed in $42.3 \%$ of the comparisons. Additionally, many of the values were between 0.45 and 0.50 (table 3 ), and therefore near the level of high similarity. Based on these results, it is possible to infer that there was considerable floristic similarity between the areas surveyed. On the other hand, all of the values generated by the Czekanowski index were low $(<0.50)$, except between Jaraguá and Mara Rosa areas (table 3) - indicating that in spite of the apparent similarities in the species compositions among the different areas, differences in relative densities that gave them unique structural characteristics. In their studies of Cerrado areas (sensu stricto) in Chapada Pratinha, Chapada dos Veadeiros and São Francisco, Felfili et al. (2004) noted that species densities were one of the most important factors in differentiating between the different areas. According to those authors, this parameter must be taken into consideration when considering strategies designed to protect populations of native woody Cerrado species. 
Table 3. Sørensen and Czekanowski floristic similarity indices, calculated based on the tree-shrub vegetation $\left(\mathrm{DB}_{30}\right.$ $\geq 5 \mathrm{~cm}$ ) surveyed in 10 areas of rocky outcrop cerrado (ten $20 \times 50 \mathrm{~m}$ plots $)$ in Goiás State, Brazil. (A1 = Alto Paraíso de Goiás; A2 = Caldas Novas; A3 = Cavalcante; A4 = Cristalina; A5 = Jaraguá; A6 = Mara Rosa; A7 = Nazário; A8 = Piranhas; A9 $=$ Pirenópolis; A10 = Mineiros). Values indicated in bold type indicate high levels of floristic similarity between the areas.

\begin{tabular}{|c|c|c|c|c|c|c|c|c|c|c|c|}
\hline & & & & & zzeka & nows & & & & & \\
\hline & & A1 & A2 & A3 & A4 & A5 & A6 & A7 & A8 & A9 & A10 \\
\hline & A1 & 1 & 0.07 & 0.15 & 0.17 & 0.11 & 0.08 & 0.10 & 0.06 & 0.19 & 0.11 \\
\hline & A2 & 0.32 & 1 & 0.21 & 0.40 & 0.35 & 0.29 & 0.40 & 0.25 & 0.21 & 0.17 \\
\hline & A3 & 0.48 & 0.47 & 1 & 0.28 & 0.35 & 0.47 & 0.29 & 0.22 & 0.20 & 0.19 \\
\hline & A4 & 0.45 & 0.61 & 0.49 & 1 & 0.29 & 0.32 & 0.30 & 0.14 & 0.39 & 0.22 \\
\hline 0 & A5 & 0.36 & 0.64 & 0.57 & 0.59 & 1 & 0.50 & 0.47 & 0.27 & 0.19 & 0.28 \\
\hline$\widetilde{\mathscr{S}}$ & A6 & 0.30 & 0.56 & 0.51 & 0.52 & 0.71 & 1 & 0.40 & 0.30 & 0.23 & 0.30 \\
\hline & A7 & 0.28 & 0.58 & 0.50 & 0.53 & 0.71 & 0.67 & 1 & 0.37 & 0.21 & 0.23 \\
\hline & A8 & 0.18 & 0.37 & 0.32 & 0.26 & 0.40 & 0.44 & 0.40 & 1 & 0.11 & 0.19 \\
\hline & A9 & 0.45 & 0.45 & 0.42 & 0.58 & 0.52 & 0.48 & 0.47 & 0.29 & 1 & 0.16 \\
\hline & A10 & 0.32 & 0.52 & 0.47 & 0.51 & 0.52 & 0.56 & 0.45 & 0.31 & 0.38 & 1 \\
\hline
\end{tabular}

The greatest similarity values in the present study were seen between Jaraguá and Mara Rosa according to both indices, and between Jaraguá and Nazário by the Sørensen index. On the other hand, the areas of Alto Paraíso de Goiás and Piranhas showed the greatest mutual differences, and were not found in the similar to any of the other survey areas as judged by either of the indices used here. Considered together, the results of the floristic similarity analyses did not reveal any relationships between the regions or with the geographical distances between the 10 sites analyzed. These results corroborated with those reported by Felfili et al. (2004), which indicated that local environmental conditions largely determine floristic similarities of cerrado (sensu stricto) areas, independent of their geographical proximity.

Similarly, the groups formed by Two-Way Indicator Species Analysis (TWINSPAN) (figure 2) (e.g. Alto Paraíso de Goiás and Pirenópolis; Cristalina and Mineiros; Caldas Novas, Jaraguá, Mara Rosa and Nazário) did not correspond to any defined geographical area, while the areas separated by the analyses were geographically close, or even belonged to the same geographical region (e.g. Alto Paraíso de Goiás and Cavalcante). As such, the results generated by TWINSPAN likewise indicated the lack of regional influences (or of the geographical distances between the areas) on their species distributions. In general, the differences in population sizes were responsible for forming these groups as the species compositions were generally homogeneous among the different areas.

The results of the Mantel test $(\mathrm{r}=0.32, P=0.05)$ likewise indicated the lack of any relationships between geographical distances separating them and the floristic similarity values of the areas. In this case, the similarities of the environmental parameters were more important in the analyses than the geographical distances between the areas, as was also observed by Conceição et al. (2007) in four areas of open rocky field vegetation in the Chapada Diamantina (Bahia State). These authors observed that the closest sites were not the most floristically similar, indicating that other factors more relevant than distance were involved in determining species compositions.

Felfili et al. (2004) compared a number of cerrado sites growing on deep soils in the Chapada Pratinha, Chapada dos Veadeiros, and Espigão Mestre do São Francisco physiographic units, and encountered high floristic similarities between them. However, when some of the sites within these units were compared with the Alto Paraíso de Goiás and Chapada dos Veadeiros sites, only low similarities were found - leading the authors to believe that the Chapada dos Veadeiros region is physiographically highly heterogeneous, and that this heterogeneity is accentuated by the presence of superficial limestone and sandstone rock formations and an irregular landscape. Additionally, Chapada dos Veadeiros is considered a center of species endemism for the Cerrado biome (Simon \& Proença 2000). These factors probably influenced the finding that there were 16 species exclusive to the Alto Paraíso de Goiás and Piranhas sites (both of which were examined in the present study). It is important to point out that the region near Piranhas has many granitic outcrops, which may have favored the establishment of these exclusive species. A number of authors have commented on the possibility that differences in environment conditions (such as edaphic and landscape qualities) could explain the low similarities seen between different areas (Felfili \& Felfili 2001, Felfili \& Silva Júnior 2005).

The peripheral geographical localization of the Alto Paraíso and Piranhas areas, together with their floristic similarities and their large numbers of exclusive species, make these areas important potential sites for the conservation of the woody rocky outcrop cerrados flora in Goiás State. According to Felfili (2007), the 


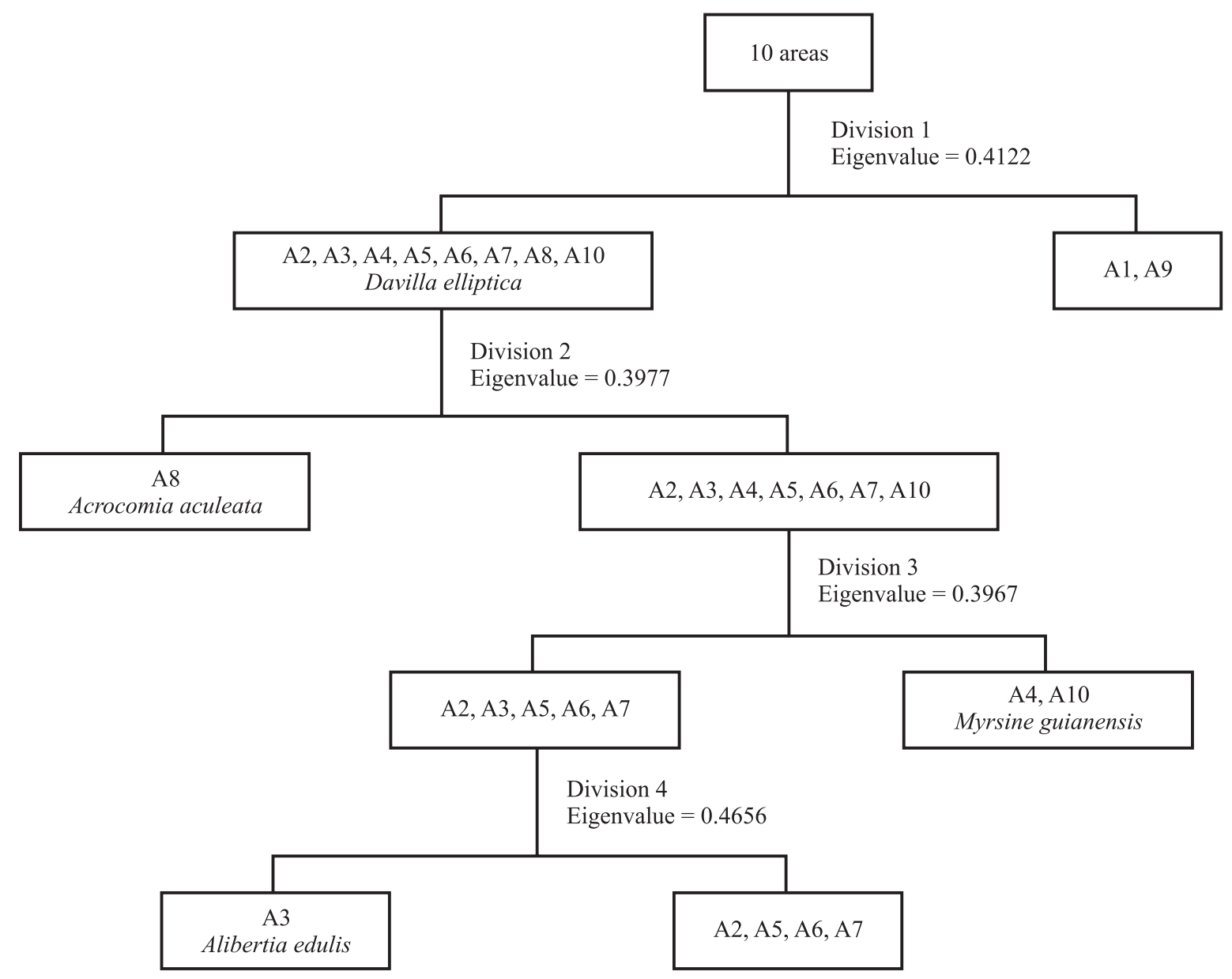

Figure 2. Dendrogram generated by divisive classification (TWINSPAN) of 10 areas of rocky outcrop cerrado surveyed in Goiás State, Brazil. (A1 = Alto Paraíso de Goiás; A2 = Caldas Novas; A3 = Cavalcante; A4 = Cristalina; A5 = Jaraguá; A6 = Mara Rosa; A7 = Nazário; A8 = Piranhas; A9 = Pirenópolis; A10 = Mineiros).

only conservation area in the region (the Chapada dos Veadeiros National Park) is not in itself sufficient to preserve the elevated richness and floristic heterogeneity found in the region. As such, we suggest the creation of new conservation areas in these two regions, especially in sites that have distinct edaphic conditions not represented in other established conservation sites.

The results of the present study indicate that the rocky outcrop cerrado vegetation of Goiás State has high floristic richness. Most of the species identified can also be found in cerrado sensu stricto sites on deep soils, and some of them are seen in forest environments, and small numbers of specialist species that inhabit specific and limited habitats. The combination of high richness, floristic uniqueness, and the small disturbance indices of rocky outcrop cerrados sites in Goiás State make them strategically valuable for the conservation of the woody vegetation of cerrado formations in the Cerrado biome.
Acknowledgments - The authors thank the Fundação Grupo Boticário de Proteção à Natureza for their financial support (Projects $n^{\circ}$ 0651/20051 and 0765/20072); Capes for awarding study grants to the first and the last authors; $\mathrm{CNPq} /$ Procad $\mathrm{UnB} /$ Unemat for financing part of the collections and the data analyses; the specialists Carolyn Proença, Lúcia Helena Soares Silva, Manoel C. da Silva Júnior, and Bruno M. T. Walter for their help in identifying the botanical material; Camila Lima for her help in the figures elaboration; and the students Thiago Abreu, Vicente Arcela, Lívia Carrera, Julianna Maroccolo, Bárbara Bomfim, Watson Rodrigues, Elisa Bruziguessi, Thaís Lima, Renato Lôbo, Ayuni Mendes, and Mariângela Abreu for their participation in the field collections.

\section{REFERENCES}

Amaral AG, Pereira FFO, Munhoz CBR. 2006. Fitossociologia de uma área de cerrado rupestre na Fazenda Sucupira, Brasília-DF. Cerne 12:350-359. 
APG III. 2009. An update of the Angiosperm Phylogeny Group classication for the orders and families of flowering plants: APG III. Botanical Journal of the Linnean Society 161:105-121.

Arruda MB, Proença CEB, Rodrigues SC, Campos RN, Martins RC, Martins ES. 2008. Ecorregiões, unidades de conservação e representatividade ecológica do bioma cerrado. In Cerrado: ecologia e flora (SM Sano, SP Almeida, JF Ribeiro, eds.). Embrapa Cerrados, Planaltina, v.1, p.265-269.

Assunção SL, Felfili JM. 2004. Fitossociologia de um fragmento de cerrado sentido restrito na APA do ParanoáDF, Brasil. Acta Botanica Brasilica18:903-909.

Benites VM, Caiafa AN, Mendonça ES, Schaefer CE, Ker JC. 2003. Solos e vegetação nos complexos rupestres de altitude da Mantiqueira e do Espinhaço. Floresta e Ambiente 10:76-85.

Benites VM, Schaefer CEGR, Simas FNB, Santos HG. 2007. Soils associated with rock outcrops in the Brazilian mountain ranges Mantiqueira and Espinhaço. Revista Brasileira de Botânica 30:569-577.

Bridgewater S, Ratter JA, Ribeiro JF. 2004. Biogeographic patterns, $\beta$ diversity and dominance in the cerrado biome of Brazil. Biodiversity and Conservation 13:2295-2318.

Brower JE, ZAR JH. 1977. Field and laboratory methods for general ecology. Wm. C. Brown Publishers, Dubuque.

Conceição AU, Pirani JR, Meirelles ST. 2007. Floristics, structure and soil of insular vegetation in four quartzitesandstone outcrops of "Chapada Diamantina", Northeast Brazil. Revista Brasileira de Botânica 30:641-656.

Eiten G. 1992. Natural Brazilian vegetation types and their causes. Anais da Academia Brasileira de Ciências 64: 35-65.

Felfili JM. 2007. Recomendações de manejo. In Biogeografia do bioma Cerrado: vegetação e solos da Chapada dos Veadeiros (JM Felfili, AV Rezende, MC Silva Júnior, orgs.). Universidade de Brasília/ Fundação de Empreendimentos Científicos e Tecnológicos - Finatec, Brasília, p.239-242.

Felfili MC, Felfili JM. 2001. Diversidade alfa e beta no cerrado sentido restrito da Chapada Pratinha, Brasil. Acta Botanica Brasilica 15:95-106.

Felfili JM, Silva Júnior MC. 2005. Diversidade alfa e beta no cerrado sensu stricto, Distrito Federal, Goiás, Minas Gerais e Bahia. In Cerrado, ecologia, biodiversidade e conservação (A Scariot, JC Sousa Silva, JM Felfili, orgs.). Ministério do Meio Ambiente, Brasília, p.143-154.

Felfili JM, Nogueira PE, Silva Júnior MC, Marimon BS, Delitti WBC. 2002. Composição florística e fitossociológica do cerrado sentido restrito no município de Água Boa-MT. Acta Botanica Brasilica 16:103-112.

Felfili JM, Silva Júnior MC, Sevilha AC, Fagg CW, Walter BMT, Nogueira PE, Rezende AV. 2004. Diversity, floristic and structural patterns of cerrado vegetation in Central Brazil. Plant Ecology 175:37-46.
Felfili JM, Carvalho FA, Haidar RF. 2005. Manual para o monitoramento de parcelas permanentes nos biomas cerrado e pantanal. Departamento de Engenharia Florestal - Universidade de Brasília, Brasília.

Forzza RC, Leitman PM, Costa AF, Carvalho Júnior AA, Peixoto AL, Walter BMT, Bicudo C, Zappi D, Costa DP, Lleras E, Martinelli G, Lima HC, Prado J, Stehmann JR, Baumgratz JFA, Pirani JR, Sylvestre L, Maia LC, Lohmann LG, Queiroz LP, Silveira M, Coelho MN, Mamede MC, Bastos MNC, Morim MP, Barbosa MR, Menezes M, Hopkins M, Secco R, Cavalcanti TB, Souza VC. 2010. Lista de espécies da flora do Brasil. Jardim Botânico do Rio de Janeiro. http://floradobrasil.jbrj.gov.br/2010/ (accessed 2010 Aug 11).

Goodland RA. 1979. Análise ecológica da vegetação do cerrado. In Ecologia do Cerrado. (RA Goodland, MG Ferri, eds.). Edusp / Itatiaia, São Paulo, p.61-171.

Haridasan M. 2000. Nutrição mineral de plantas nativas do cerrado. Revista Brasileira de Fisiologia Vegetal 12: 54-64.

Kent M, Coker P. 1992. Vegetation description and analysis: a practical approach. Bealhaven Press, London.

Legendre P, Legendre L. 1998. Numerical ecology, Elsevier Science B.V., Amsterdam.

Lenza E, Pinto JRR, Pinto AS, Maracahipes L, Bruziguessi EL. 2011. Comparação da vegetação arbustivo-arbórea de uma área de cerrado rupestre na Chapada dos Veadeiros, Goiás e áreas de cerrado sentido restrito do Bioma Cerrado. Revista Brasileira de Botânica 34: 247-259.

Lima CA. 2008. O cerrado rupestre no Estado de Goiás com base em imagens LANDSAT +. Dissertação de mestrado, Universidade de Brasília, Brasília.

Lima TA, Pinto JRR, Lenza E, Pinto AS. 2010. Florística e estrutura da vegetação arbustivo-arbórea em uma área de cerrado rupestre no Parque Estadual da Serra de Caldas Novas, Goiás. Biota Neotropica 10:159-166.

Manoel LC. 1999. Composição florística, fitossociológica e estado nutricional de comunidades arbóreas de um cerrado rupestre e um cerrado ralo na Serra DouradaGO. Dissertação de mestrado, Universidade Federal de Goiás, Goiânia.

Marimon BS, Varella RF, Marimon Junior BH. 1998. Fitossociologia de uma área de cerrado de encosta em Nova Xavantina, Mato Grosso. Boletim do Herbário Ezechias Paulo Heringer 3:82-101.

McCune B, Mefford MJ. 1999. PCORD: multivariate analysis of ecological data. Version 4.0 MjM Software Design, Gleneden Beach.

Mendonça RC, Felfili JM, Walter BMT, Silva Júnior MC, Rezende AV, Filgueiras TS, Nogueira PE. 2008. Flora vascular do cerrado. In Cerrado: ecologia e flora (SM Sano, SP Almeida, eds.). Embrapa Cerrados, Planaltina, v.2, p.1:1279. 
Miranda SC, Silva Júnior MC, Salles LAA. 2007. Comunidade lenhosa de cerrado rupestre na Serra Dourada, Goiás. Heringeriana 1:43-53.

Moura IO, Gomes Klein VL, Felfili JM, Ferreira HD. 2007. Fitossociologia de cerrado sentido restrito em afloramentos rochosos no Parque Estadual dos Pireneus, Pirenópolis, Goiás. Revista Brasileira de Biociências 5:399-401.

Moura IO, Klein VLG, Felfili JF, Ferreira HD. 2010. Diversidade e estrutura comunitária de cerrado sentido restrito em afloramentos rochosos no Parque Estadual dos Pireneus, Goiás. Revista Brasileira de Botânica 33:55-467.

Moura IO. 2010. Fitogeografia do cerrado rupestre: relações florístico-estruturais e ecológicas de espécies lenhosas. Tese de doutorado, Universidade de Brasília, Brasília.

Munhoz CBR, Proença CEB. 1998. Composição florística do município de Alto Paraíso de Goiás na Chapada dos Veadeiros. Boletim do Herbário Ezechias Paulo Heringer 3:102-150.

Oliveira Filho AT, Ratter JA. 2002. Vegetation physiognomies and woody flora of the Cerrado biome. In The Cerrados of Brazil (PS Oliveira, RJ Marquis, eds.). Columbia University Press, New York, p.91-120.

Pinto JRR, Lenza E, Pinto AS. 2009. Composição florística e estrutura da vegetação arbustivo-arbórea em um Cerrado Rupestre, Cocalzinho de Goiás, GO. Revista Brasileira de Botânica 32:1-10.

Rabinowits D. 1981. Seven forms of rarity. In The biological aspects of rare plant conservation (H Synge, ed.). Jonh Wiley, Chicester, p.205-217.

Ratter JA, Bridgewater S, Ribeiro JF, Dias TAB, Silva MR. 2000. Estudo preliminar da distribuição das espécies lenhosas da fitofisionomia cerrado sentido restrito nos Estados compreendidos pelo bioma Cerrado. Boletim do Herbário Ezechias Paulo Heringer 5:5-43.

Ratter JA, Bridgewater S, Ribeiro JF. 2003. Analysis of the floristic composition of the Brazilian cerrado vegetation III: comparison of the woody vegetation of 376 areas. Edinburgh Journal of Botany 60:57-109.
Reatto A, Correia JR, Spera ST, Martins ES. 2008. Solos do Bioma do Cerrado: aspectos pedológicos. In Cerrado: ecologia e flora (SM Sano, SP Almeida, eds.). Embrapa Cerrados, Planaltina, v.1, p.151-199.

Ribeiro JF, Walter BMT. 2008. Fitofisionomias do bioma Cerrado. In Cerrado: ecologia e flora (SM Sano, SP Almeida, eds.). Embrapa Cerrados, Planaltina, v.1, p.89-168.

Romero R. 2002. Diversidade da flora dos campos rupestres de Goiás, Sudoeste e Sul de Minas Gerais. In Biodiversidade, conservação e uso sustentável da flora do Brasil (EL Araújo, AN Moura, ESB Sampaio, LMS Gestinari, JMT Carneiro, eds.). Universidade Federal Rural de Pernambuco / Sociedade Botânica do Brasil, Recife, p.81-86.

Sano EE, Dambrós LA, Oliveira GC, Brites RS. 2006. Padrões de cobertura de solos do Estado de Goiás. In Conservação da biodiversidade e sustentabilidade ambiental em Goiás: prioridades, estratégias e perspectivas (LG Ferreira Júnior, Org.). Universidade Federal de Goiás/Agência Ambiental/Banco Mundial, Goiânia, p.76-93.

Sano EE, Rosa R, Brito JLS, Ferreira LG, Bezerra HS. 2009. Mapeamento da cobertura vegetal natural e antrópica do bioma Cerrado por meio de imagens Landsat ETM+. In Anais do Simpósio Brasileiro de Sensoriamento Remoto. INPE, Natal, p.1199-1206.

SIEG - Sistema Estadual de Estatística e de Informações Geográficas de Goiás. 2009. Brasília-DF. http://www. sieg.go.gov.br. (accessed 2009 Jun 02).

Silva SR, Silva AP, Munhoz CB, Silva Júnior MC, Medeiros MB. 2001. Guia de plantas do Cerrado utilizadas na Chapada dos Veadeiros. WWF - Brasil, Brasília.

Simon MF, Proença C. 2000. Phytogeographic patterns of Mimosa (Mimosoideae, Leguminosae) in the Cerrado biome of Brazil: an indicator genus of high-altitude center of endemism? Biological Conservation 96:279-296.

Van den Berg E, Oliveira Filho AT. 2001. Composição florística e estrutura fitossociológica de uma floresta ripária em Itutinga, MG, e comparação com outras áreas. Revista Brasileira de Botânica 23:231-253. 